\title{
Modelagem Direta Ótima de dados mCSEM 2.5D de Ambientes Resistivos em Águas Profundas
}

Frayzer L. de Almeida(1)

(1) Campus de Castanhal/UFPA

Copyright 2021, SBGf - Sociedade Brasileira de Geofísica.

This paper was prepared for presentation during the $17^{\text {th }}$ International Congress of the Brazilian Geophysical Society held in Rio de Janeiro, Brazil, 16-19 August 2021.

Contents of this paper were reviewed by the Technical Committee of the $17^{\text {th }}$ International Congress of the Brazilian Geophysical Society and do 17 International Congress of the Brazilian Geophyscal Society and do not necessarily represent any position of the SBG, its officers or members. Electronic reproduction or storage of any part of this paper for commercial purposes without the written consent of the Brazilian

\section{Abstract}

We propose optimal numerical solutions for the direct problem of mCSEM 2.5D using the twodimensional finite element method. To this end, we developed strategies to minimize the processing time for these solutions. We evaluated the validation and / or calibration of the mCSEM 2.5D modeling based on comparisons between mCSEM 2.5D and 1D solutions. We verified the sensitivity of mCSEM 2.5D solutions using geoelectric models of mCSEM 2.5D proposed by Rijo (2005).

\section{Resumo}

Propomos soluções numéricas ótimas para o problema direto do mCSEM 2.5D através do método dos elementos finitos bidimensionais. Para tanto, desenvolvemos estratégias para minimizar o tempo de processamento destas soluções. Avaliamos a validação e/ou a calibração da modelagem do mCSEM 2.5D a partir de comparações entre soluções do mCSEM 2.5D e 1D. Verificamos a sensibilidade das soluções do mCSEM 2.5D através de modelos geoelérticos do mCSEM 2.5D propostos por Rijo (2005).

\section{Introdução}

Desde 2000 os cenários na exploração de hidrocarbonetos vêm mudando. No sentido de auxiliar a sísmica na detecção de hidrocarbonetos e minimizar o risco exploratório faz-se necessário a incursão de técnicas nãosísmicas. Também, o campo para a exploração de hidrocarbonetos se expandiu para regiões de águas profundas e ultra-profundas em ambientes altamente resistivos, onde o preço de um poço exploratório de petróleo é exorbitantemente alto (Rijo, 2007). Dessa forma, os métodos geofísicos elétricos e eletromagnéticos - EM apropriados e indicados na prospecção de hidrocarbonetos em águas profundas e ultra-profundas são o método Eletromagnético Marinho de Fonte Controlada mCSEM e o método Magnetotelúrico Marinho MMT. São métodos que vêm se destacando na prospecção de hidrocarbonetos em águas profundas e ultra-profundas nas últimas duas décadas, pois usados em conjunto à sísmica podem confirmar a existência de hidrocarbonetos, mapear o contorno de um reservatório e contribuir para a diminuição da ambigüidade na interpretação geológica.

O mCSEM usa como fonte, um dipolo elétrico horizontal (DEH) móvel próximo ao assoalho oceânico, e um arranjo de receptores de campos eletromagnéticos que se encontram depositados também no assoalho oceânico como, por exemplo, em bacias costeiras no Brasil. O dipolo elétrico transmite um sinal eletromagnético de baixa frequência, de décimos à algumas unidades de Hertz, energizando as camadas sedimentares a partir do fundo do mar. Devido a natureza tridimensional da fonte, a difusão da energia eletromagnética (EM) se dá em todas as direções da coluna de água e o sinal EM detectado nos arranjos de receptores é uma combinação da energia EM transmitida diretamente entre o transmissor e o receptor e da energia EM refletidos e refratados pelo mar, pelo ar e pelas camadas de interesse abaixo do assoalho oceânico, ou seja, reservatórios de hidrocarbonetos (Souza, 2007).

Em Rijo (2005) foi desenvolvido um algoritmo segundo a modelagem de dados $2.5 \mathrm{D}$ do mCSEM através do método dos elementos finitos bidimensionais, o qual apresenta o tempo de duração de processamento de aproximadamente 3 minutos, em um PC Pentium IV, quando aplicado sobre modelos geoelétricos do mCSEM 2.5D. A partir daí, propomos um algoritmo ótimo sobre dados $2.5 \mathrm{D}$ do mCSEM. Para tanto, primeiramente desenvolvemos a modelagem direta de dados 2.5D do mCSEM através do método dos elementos finitos bidimensionais. Em segundo, discutimos, analisamos e tomamos decisões estratégicas no sentido de minimizar o tempo de processamento das simulações computacionais de dados 2.5D do mCSEM. Em terceiro, discutimos através de resultados e/ou soluções segundo modelos geoelétricos do mCSEM 2.5D e 1D sobre a calibração e/ou validação da modelagem 2.5D acima proposta. Finalmente, analisamos os resultados, através do algoritmo proposto, segundo modelos geolétricos do mCSEM 2.5D propostos por Rijo (2005), no sentido de verificar a sensibilidade destes resultados e/ou soluções em relação à variação do comprimento das heterogeneidades, com hidrocarbonetos, presentes nos modelos geolétricos do mCSEM 2.5D em Rijo (2005).

\section{Problema direta do método mCSEM 2.5D}

A partir das equações de Maxwell (no sistema SI) no domínio da freqüência e em um meio não 
magnético, podemos expressar os campos secundários elétrico $\mathbf{E}^{\mathrm{s}}$ e magnético $\mathbf{H}^{\mathrm{s}}$ na seguinte forma:

$$
\begin{aligned}
& \boldsymbol{\nabla} \times \mathbf{H}^{\mathrm{s}}-\sigma \mathbf{E}^{s}=\Delta \boldsymbol{\sigma} \mathbf{E}^{p} \\
& \boldsymbol{\nabla} \times \mathbf{E}^{s}+i \omega \mu \mathbf{H}^{s}=\mathbf{0},
\end{aligned}
$$

em que $\sigma$ e $\sigma^{\mathrm{p}}$ são a condutividade elétrica da heterogeneidade e a condutividade do meio homogêneo, respectivamente, tal que $\Delta \sigma=\sigma-$ $\sigma^{p}$, a permeabilidade magnética do ar $\boldsymbol{\mu}=$ $4 \pi 10^{-7} \mathrm{H} / \mathrm{m}, \omega=2 \pi f$ a frequência angular, $f$ a frequência linear, e $\mathbf{E}^{p}$ o campo elétrico primário do meio homogêneo (Rijo, 2002).

A primeira equação, Eq.(1), expressa a partir de suas componentes $x, y$ e $z$, fica:

$$
\begin{aligned}
& \frac{\partial H_{z}^{s}}{\partial y}-\frac{\partial H_{y}^{s}}{\partial z}-\sigma E_{x}^{s}=\nabla \sigma E_{x}^{p}, \\
& \frac{\partial H_{x}^{s}}{\partial z}-\frac{\partial H_{z}^{s}}{\partial x}-\sigma E_{y}^{s}=\nabla \sigma E_{y}^{p}, \\
& \frac{\partial H_{y}^{s}}{\partial x}-\frac{\partial H_{x}^{s}}{\partial y}-\sigma E_{z}^{s}=\nabla \sigma E_{z}^{p} .
\end{aligned}
$$

Já a segunda equação, Eq.(2), expressa a partir de suas componentes $x$, y e $z$, fica:

$$
\begin{aligned}
& \frac{\partial E_{z}^{s}}{\partial y}-\frac{\partial E_{y}^{s}}{\partial z}+i \omega \mu H_{x}^{s}=0, \\
& \frac{\partial E_{x}^{s}}{\partial z}-\frac{\partial E_{z}^{s}}{\partial x}+i \omega \mu H_{y}^{s}=0, \\
& \frac{\partial E_{y}^{s}}{\partial x}-\frac{\partial E_{x}^{s}}{\partial y}+i \omega \mu H_{z}^{s}=0
\end{aligned}
$$

Agora, considerando que na direção do eixo $y$ as propriedades físicas não variam, aplicamos a transformada de Fourier sobre as Eqs.(3)-(5) e obtemos, no domínio $\left(\mathrm{x}, \mathrm{k}_{\mathrm{y}}, \mathrm{z}\right)$, as seguintes equações diferenciais:

$$
\begin{gathered}
i k_{y} \widehat{H}_{z}^{s}-\frac{\partial \widehat{H}_{y}^{s}}{\partial z}-\sigma \hat{E}_{x}^{s}=\Delta \sigma \hat{E}_{x}^{p}, \\
\frac{\partial \widehat{H}_{x}^{s}}{\partial z}-\frac{\partial \widehat{H}_{z}^{s}}{\partial x}-\sigma \widehat{E}_{y}^{s}=\Delta \sigma \hat{E}_{y}^{p}, \\
\frac{\partial \widehat{H}_{y}^{s}}{\partial x}-i k_{y} \widehat{H}_{x}^{s}-\sigma \hat{E}_{z}^{s}=\Delta \sigma \hat{E}_{z}^{p} .
\end{gathered}
$$

Da mesma forma, ao aplicarmos a transformada de Fourier sobre as Eqs.(6)-(8), obtemos, também no domínio $\left(\mathrm{x}, \mathrm{k}_{\mathrm{y}}, \mathrm{z}\right)$, as seguintes equações diferenciais:

$$
\begin{aligned}
& i k_{y} \hat{E}_{z}^{s}-\frac{\partial \hat{E}_{y}^{s}}{\partial z}+i \omega \mu \widehat{H}_{x}^{s}=0 \\
& \frac{\partial \widehat{E}_{x}^{s}}{\partial z}-\frac{\partial \widehat{E}_{z}^{s}}{\partial x}+i \omega \mu \widehat{H}_{y}^{s}=0 \\
& \frac{\partial \hat{E}_{y}^{s}}{\partial x}-i k_{y} \widehat{E}_{x}^{s}+i \omega \mu \widehat{H}_{z}^{s}=0
\end{aligned}
$$

Dado que as componentes $\hat{E}_{y}^{s}$ e $\widehat{H}_{y}^{s}$ são linearmente independentes então, em linhas gerais, vamos expressar as componentes $\hat{E}_{x}^{s}$,
$\widehat{E}_{z}^{s}, \widehat{H}_{x}^{s}$ e $\widehat{H}_{z}^{s}$ em função de $\widehat{E}_{y}^{s}$ e $\widehat{H}_{y}^{s}$, e também com as componentes do campo elétrico primário. Assim, substituindo a Eq.(14) na Eq.(9), obtemos a componente $\hat{E}_{x}^{s}$ :

$$
\hat{E}_{x}^{s}=-\frac{i k_{y}}{u^{2}} \frac{\partial \hat{E}_{y}^{s}}{\partial x}-\frac{i \omega \mu}{u^{2}} \frac{\partial \widehat{H}_{y}^{s}}{\partial z}-\frac{i \omega \mu}{u^{2}} \Delta \sigma \hat{E}_{x}^{p},
$$

em seguida, substituindo a Eq. (12) na Eq.(11), obtermos a componente $\hat{E}_{z}^{s}$ :

$$
\hat{E}_{z}^{s}=-\frac{i k_{y}}{u^{2}} \frac{\partial \hat{E}_{y}^{s}}{\partial z}+\frac{i \omega \mu}{u^{2}} \frac{\partial \widehat{H}_{y}^{s}}{\partial x}-\frac{i \omega \mu}{u^{2}} \Delta \sigma \hat{E}_{z}^{p}
$$

agora, substituindo a Eq.(16) na Eq. (11), obtemos a componente $\widehat{H}_{x}^{s}$ :

$$
\widehat{H}_{x}^{s}=\frac{\sigma}{u^{2}} \frac{\partial \hat{E}_{y}^{s}}{\partial z}-\frac{i k_{y}}{u^{2}} \frac{\partial \widehat{H}_{y}^{s}}{\partial x}+\frac{i k_{y}}{u^{2}} \Delta \sigma \hat{E}_{z}^{p},
$$

e, substituindo a Eq.(15) na Eq. (14), obtemos a componente $\widehat{H}_{z}^{s}$ :

$$
\widehat{H}_{z}^{s}=-\frac{\sigma}{u^{2}} \frac{\partial \hat{E}_{y}^{s}}{\partial x}-\frac{i k_{y}}{u^{2}} \frac{\partial \widehat{H}_{y}^{s}}{\partial z}-\frac{i k_{y}}{u^{2}} \Delta \sigma \hat{E}_{x}^{p},
$$

sendo a variável $u$ a constante de propagação de onda no domínio $\left(\mathrm{x}, \mathrm{k}_{\mathrm{y}}, \mathrm{z}\right)$ que é dada por $u^{2}=$ $k_{y}^{2}+i \omega \mu \sigma$.

Agora, substituímos as componentes $\widehat{H}_{x}^{s}$ e $\widehat{H}_{z}^{s}$ na Eq.(10) e as componentes $\hat{E}_{x}^{s}$ e $\hat{E}_{z}^{s}$ na Eq.(13), obtendo assim dois sistemas de equações diferencias parciais em termos das componentes $\widehat{E}_{y}^{s}$ e $\widehat{H}_{y}^{s}$, (Silva, 2012), o qual é solucionado pelo método dos elementos finitos bidimensionais (Rijo, 2005):

$$
\begin{aligned}
& -\frac{\partial}{\partial x}\left(\frac{\sigma}{u^{2}} \frac{\partial \hat{E}_{y}^{s}}{\partial x}\right)+\sigma \hat{E}_{y}^{s}-\frac{\partial}{\partial z}\left(\frac{\sigma}{u^{2}} \frac{\partial \hat{E}_{y}^{s}}{\partial z}\right)-\frac{\partial}{\partial x}\left(\frac{i k_{y}}{u^{2}} \frac{\partial \widehat{H}_{y}^{s}}{\partial z}\right)+ \\
& \frac{\partial}{\partial z}\left(\frac{i k_{y}}{u^{2}} \frac{\partial \widehat{H}_{y}^{s}}{\partial x}\right)=\frac{\partial}{\partial x}\left(\frac{i k_{y}}{u^{2}} \Delta \sigma \hat{E}_{x}^{p}\right)-\Delta \sigma \hat{E}_{y}^{p} \\
& +\frac{\partial}{\partial z}\left(\frac{i k_{y}}{u^{2}} \Delta \sigma \hat{E}_{z}^{p}\right) \\
& \frac{\partial}{\partial x}\left(\frac{i k_{y}}{u^{2}} \frac{\partial \hat{E}_{y}^{s}}{\partial z}\right)-\frac{\partial}{\partial z}\left(\frac{i k_{y}}{u^{2}} \frac{\partial \hat{E}_{y}^{s}}{\partial x}\right)-\frac{\partial}{\partial x}\left(\frac{i \omega \mu}{u^{2}} \frac{\partial \widehat{H}_{y}^{s}}{\partial x}\right)+i \omega \mu \widehat{H}_{y}^{s}- \\
& \frac{\partial}{\partial z}\left(\frac{i \omega \mu}{u^{2}} \frac{\partial \widehat{H}_{y}^{S}}{\partial z}\right)=\frac{\partial}{\partial z}\left(\frac{i \omega \mu}{u^{2}} \Delta \sigma \hat{E}_{x}^{p}\right) \\
& -\frac{\partial}{\partial x}\left(\frac{i \omega \mu}{u^{2}} \Delta \sigma \hat{E}_{z}^{p}\right)
\end{aligned}
$$

em que as duas últimas parcelas no primeiro membro da Eq.(19), e as duas primeiras parcelas no primeiro membro da Eq.(20), representam e/ou constam na caracterização do acoplamento entre os modos de propagação TE e TM.

Assim, considerando um elemento genérico (e/ou domínio genérico bidimensional) $\Omega_{e}$ da malha de elementos finitos bidimensionais e empregando o método de Galerkin às Eqs.(19) e (20) e, em seguida, usando as identidades da derivada do produto entre as funções bases, $\varphi_{m}$, e os campos eletromagnéticos, juntamente com a aplicação do teorema da divergência no plano (Jin, 2002) é possível 
expressar ambas as Eqs. (19) e (20) da seguinte forma:

$$
\begin{aligned}
& \frac{\sigma_{e}}{u_{e}^{2}} \int_{\Omega_{e}}\left(\frac{\partial \varphi_{m}}{\partial x} \frac{\partial \hat{E}_{y}^{s}}{\partial x}+\frac{\partial \varphi_{m}}{\partial z} \frac{\partial \hat{E}_{y}^{s}}{\partial z}\right) d x d z+\sigma_{e} \int_{\Omega_{e}} \varphi_{m} \hat{E}_{y}^{s} d x d z \\
& +\frac{i k_{y}^{e}}{u_{e}^{2}} \int_{\Omega_{e}}\left(\frac{\partial \varphi_{m}}{\partial x} \frac{\partial \hat{H}_{y}^{s}}{\partial z}-\frac{\partial \varphi_{m}}{\partial z} \frac{\partial \hat{H}_{y}^{s}}{\partial x}\right) d x d z= \\
& -\frac{i k_{y}^{e}}{u_{e}^{2}} \Delta \sigma_{e} \int_{\Omega_{e}}\left(\frac{\partial \varphi_{m}}{\partial x} \hat{E}_{x}^{p}+\frac{\partial \varphi_{m}}{\partial z} \hat{E}_{z}^{p}\right) d x d z- \\
& \quad \Delta \sigma_{e} \int_{\Omega_{e}} \varphi_{m} \hat{E}_{y}^{p} d x d z+\int_{\partial \Omega_{e}} \varphi_{m} \hat{A} \cdot \hat{n}_{e} d \partial \Omega_{e} \\
& -\frac{i k_{y}^{e}}{u_{e}^{2}} \int_{\Omega_{e}}\left(\frac{\partial \varphi_{m}}{\partial x} \frac{\partial \hat{E}_{y}^{s}}{\partial z}-\frac{\partial \varphi_{m}}{\partial z} \frac{\partial \hat{E}_{y}^{s}}{\partial x}\right) d x d z+\frac{i \omega \mu}{u_{e}^{2}} \int_{\Omega_{e}}\left(\frac{\partial \varphi_{m}}{\partial x} \frac{\partial \hat{H}_{y}^{s}}{\partial x}+\right. \\
& \left.\frac{\partial \varphi_{m}}{\partial z} \frac{\partial \hat{H}_{y}^{s}}{\partial z}\right) d x d z+i \omega \mu \int_{\Omega_{e}} \varphi_{m} \widehat{H}_{y}^{s} d x d z= \\
& -\frac{i \omega \mu}{u_{e}^{2}} \Delta \sigma_{e} \int_{\Omega_{e}}\left(\frac{\partial \varphi_{m}}{\partial z} \hat{E}_{x}^{p}-\frac{\partial \varphi_{m}}{\partial x} \hat{E}_{z}^{p}\right) d x d z+\int_{\partial \Omega_{e}} \varphi_{m} \hat{B} . \\
& \hat{n}_{e} d \partial \Omega_{e} .
\end{aligned}
$$

Em que $\hat{A}=\frac{i k_{y}^{e}}{u_{e}^{2}} \Delta \sigma_{e} \hat{E}_{x}^{p} \hat{x}+\frac{i k_{y}^{e}}{u_{e}^{2}} \Delta \sigma_{e} \hat{E}_{z}^{p} \hat{z} \quad$ e $\hat{B}=$ $-\frac{i \omega \mu}{u_{e}^{2}} \Delta \sigma_{e} \hat{E}_{z}^{p} \hat{x}+\frac{i \omega \mu}{u_{e}^{2}} \Delta \sigma_{e} \hat{E}_{x}^{p} \hat{z}$. Vale ressaltar que integrais de linha como, por exemplo, as que podem ser observadas nas últimas parcelas do segundo membro das Eqs. (21) e (22), respectivamente, também surgem nos primeiros membros destas equações, porém não às expressamos, no entanto mais adiante iremos justificar isto.

Para um elemento triangular genérico, as funções bases são:

$$
\begin{aligned}
& \varphi_{m}=\frac{1}{2 s_{e}}\left(a_{m}+b_{m} x+c_{m} z\right) \text { e } \varphi_{n}=\frac{1}{2 s_{e}}\left(a_{n}+\right. \\
& \left.b_{n} x+c_{n} z\right) \\
& \text { com os índices }(m=1,3) \text { e }(n=1,3)
\end{aligned}
$$

(Zienkiewicz; Taylor, 2000), onde $S_{e}$ é área do triângulo genérico e $a_{m}, b_{m}, c_{m}, a_{n}, b_{n}, c_{n}$ são constantes a serem determinadas em termos das coordenadas $\mathrm{x}$ e $\mathrm{z}$ de cada vértice do triângulo genérico. Assim temos:

$$
\hat{E}_{y}^{s}=\sum_{n=1}^{3} \hat{E}_{y, n}^{s} \varphi_{n} \text { e } \widehat{H}_{y}^{s}=\sum_{n=1}^{3} \widehat{H}_{y, n}^{s} \varphi_{n}
$$

o que é análogo para as componentes $\widehat{E}_{x}^{p}, \hat{E}_{y}^{p} \mathrm{e}$ $\hat{E}_{z}^{p}$, também em função de $\varphi_{n}$.

Agora, expandindo a componente magnética $\widehat{H}_{y}^{s}$ e as componentes elétricas $\hat{E}_{y}^{s}, \hat{E}_{x}^{p}, \hat{E}_{y}^{p}$ e $\hat{E}_{z}^{p}$, referentes às Eqs. (21) e (22), sendo essas expansões em termos das funções bases, $\varphi_{n}$, para um triângulo (Zienkiewicz; Taylor, 2000), onde $(n=1,3)$, juntamente com as aplicações das condições de continuidade dos campos eletromagnéticos primários e secundários, e finalmente com a substituição de $\varphi_{m}$ e $\varphi_{n}$ nas equações advindas das Eqs. (21) e (22) e considerando as propriedades elétricas $(\mu, \sigma)$ constantes no interior de cada elemento triangular, são obtidos os seguintes sistemas de equações lineares locais para um elemento triangular genérico:

$$
\begin{aligned}
& \sum_{n=1}^{3} \hat{E}_{y, n}^{s}\left[\frac{\sigma_{e}}{4 S_{e} u_{e}^{2}}\left(b_{m} b_{n}+c_{m} c_{n}\right)+\frac{\sigma_{e} S_{e}}{12}\left(1+\delta_{m n}\right)\right]+ \\
& \sum_{n=1}^{3} \widehat{H}_{y, n}^{s}\left[\frac{i k_{y}^{e}}{4 S_{e} u_{e}^{2}}\left(b_{m} c_{n}-c_{m} b_{n}\right)\right]= \\
& {\left[\left(-\frac{i k_{y}^{e}}{6 u_{e}^{2}} \Delta \sigma_{e}\right)\left(\left(b_{m} \sum_{n=1}^{3} \hat{E}_{x, n}^{p}\right)+\left(c_{m} \sum_{n=1}^{3} \hat{E}_{z, n}^{p}\right)\right)\right]-} \\
& \sum_{n=1}^{3} \widehat{E}_{y, n}^{p}\left[\frac{\Delta \sigma_{e} S_{e}}{12}\left(1+\delta_{m n}\right)\right] \\
& \sum_{n=1}^{3} \hat{E}_{y, n}^{s}\left[-\frac{i k_{y}^{e}}{4 S_{e} u_{e}^{2}}\left(b_{m} c_{n}-c_{m} b_{n}\right)\right]+ \\
& \sum_{n=1}^{3} \widehat{H}_{y, n}^{s}\left[\frac{i \omega \mu}{u_{e}^{2}}\left(b_{m} b_{n}+c_{m} c_{n}\right)+\frac{i \omega \mu S_{e}}{12}\left(1+\delta_{m n}\right)\right]= \\
& {\left[\left(-\frac{i \omega \mu}{6 u_{e}^{2}} \Delta \sigma_{e}\right)\left(\left(c_{m} \sum_{n=1}^{3} \hat{E}_{x, n}^{p}\right)-\left(b_{m} \sum_{n=1}^{3} \hat{E}_{z, n}^{p}\right)\right)\right]}
\end{aligned}
$$

Observamos que as integrais de linha, existentes nas Eqs.(21) e (22), não aparecem nas Eqs. (23) e (24), uma vez que elas cancelam-se entre os elementos triangulares internos, isto ocorre graças à continuidade tangencial das componentes dos campos elétricos, magnéticos e também das funções bases. Por outro lado, na borda da malha de elementos finitos, estas integrais de linha não precisam ser calculadas, pois aplicamos a condição de contorno homogênea de Dirichlet no bordo da referida malha. A partir das Eqs.(23) e (24), em um elemento triangular genérico, obtemos o sistema linear local de ordem $6 \times 6$, e de posse das componentes primárias do campo elétrico, também no domínio $(\mathrm{x}, \mathrm{ky}, \mathrm{z})$, isto é, $\hat{E}_{x}^{p}, \hat{E}_{y}^{p}$ e $\hat{E}_{z}^{p}$, obtemos o sistema linear global associado a uma matriz complexa, simétrica e esparsa. Este sistema é solucionado numericamente pelo método do gradiente biconjugado (Souza et al., 2005). Depois de solucionar o sistema global de equações lineares para as componentes secundárias, $\widehat{E}_{y}^{s}$ e $\widehat{H}_{y}^{s}$, no domínio $\left(\mathrm{x}, \mathrm{k}_{\mathrm{y}}, \mathrm{z}\right)$, calculamos as componentes também secundárias, $\hat{E}_{x}^{s}$ e $\hat{E}_{z}^{s}$, também no domínio $(\mathrm{x}, \mathrm{ky}, \mathrm{z})$, usando as Eqs.(15) e (16), respectivamente. Em seguida aplicamos a transformada inversa de Fourier no domínio (x,ky,z), sobre as componentes $\hat{E}_{x}^{s}, \hat{E}_{z}^{s}, \hat{E}_{x}^{p}$ e $\hat{E}_{z}^{p}$, obtendo assim as seguintes componentes $E_{x}^{S}$ , $E_{z}^{s}, E_{x}^{p}$ e $E_{z}^{p}$, e consequentemente os campos elétricos totais das componentes $x$ e $z$, respectivamente.

A formulação das componentes $\hat{E}_{x}^{p}, \hat{E}_{y}^{p}$ e $\hat{E}_{z}^{p}$ constam em Rijo (2002), Souza et al. (2007) e em Silva (2012). Como a componente $\hat{E}_{x}^{p}$ é função par, na variável kx, e as componentes $\hat{E}_{y}^{p}$ e $\hat{\hat{E}}_{z}^{p}$ são funções ímpares, também na variável kx (Silva, 2012), portanto basta aplicar, sobre a primeira componente a transformada cosseno, e sobre as duas últimas a transformada seno, no domínio $\left(\mathrm{k}_{\mathrm{x}}, \mathrm{k}_{\mathrm{y}}, \mathrm{z}\right)$, mas sobre a variável $\mathrm{k}_{\mathrm{x}}$, obtendo assim as componentes $\hat{E}_{x}^{p}, \hat{E}_{y}^{p}$ e $\hat{E}_{z}^{p}$ no domínio $\left(\mathrm{x}, \mathrm{k}_{\mathrm{y}}, \mathrm{z}\right)$. Estas últimas componentes são obtidas pelo método numérico da Quadratura Gaussiana (Burden, 2008). 
Estratégias para minimizar o tempo de processamento do mCSEM 2.5D

Métodos eletromagnéticos, como este do mCSEM 2.5D, precisam calcular suas componentes primárias, que compõe a fonte, centenas e/ou milhares de vezes durante o seu processamento, uma vez que elas são de fundamental importância para os métodos eletromagnéticos que não possuem soluções analíticas. Em geral estas componentes primárias também não têm soluções analíticas, tornando o processamento dos métodos eletromagnéticos bem dispendiosos em relação ao tempo, uma vez que estas componentes primárias são calculadas durante o processo através de transformadas numéricas. Portanto, no sentido de minimizar o tempo de processamento das simulações computacionais do método mCSEM 2.5D, vamos discutir, analisar e tomar decisões pragmáticas acerca das componentes primárias $\hat{E}_{x}^{p}, \hat{E}_{y}^{p}$ e $\hat{E}_{z}^{p}$ do referido método eletromagnético.

Observamos primeiramente nas Eqs.(23) e (24), que as componentes do campo elétrico primário existem apenas na heterogeneidade, uma vez que nela temos $\Delta \sigma_{e} \neq 0$, já fora da heterogeneidade temos que $\Delta \sigma_{e}=0$, daí estas componentes do campo elétrico primário são anuladas, ou seja, basta calcular as componentes $\hat{E}_{x}^{p}, \hat{E}_{y}^{p}$ e $\hat{E}_{z}^{p}$ apenas nos nós e/ou nos vértices dos triângulos pertencentes no interior e na borda da heterogeneidade. Em segundo para obtermos as componentes totais $E_{x}$ e $E_{z}$, existentes no assoalho oceânico, às quais são medidas em receptores, precisamos das componentes $E_{x}^{p}, E_{x}^{s}, E_{z}^{p}$ e $E_{z}^{s}$, também no assoalho oceânico, sendo que as componentes secundárias $E_{x}^{S}$ e $E_{Z}^{S}$ são calculadas através da resolução do sistema linear global em conjunto com procedimentos metodológicos dos elementos finitos bidimensionais já descritos, por outro lado as componentes primárias $E_{x}^{p} \mathrm{e}$ $E_{z}^{p}$ são obtidas pela transformada cosseno sobre as componentes primárias $\hat{E}_{x}^{p}$ e $\hat{E}_{z}^{p}$ existentes no assoalho oceânico, uma vez que estas últimas são funções pares. Em resumo, precisamos, para obtermos a solução do método mCSEM 2.5D, $E_{x}$ e $E_{z}$, apenas das componentes $\hat{E}_{x}^{p}, \hat{E}_{y}^{p}$ e $\hat{E}_{z}^{p}$ na heterogeneidade e das componentes $\hat{E}_{x}^{p}$ e $\hat{E}_{z}^{p}$ no assoalho oceânico, em função de cada uma das 40 abscissas do filtro linear digital da transformada cosseno (Almeida, 2002), sobre o domínio ky.

Assim, sabendo da localização, na malha de elementos finitos, das componentes primárias, $\hat{E}_{x}^{p}, \hat{E}_{y}^{p}$ e $\hat{E}_{z}^{p}$, às quais são suficientes para obtermos a solução numérica do método mCSEM 2.5D, então optamos por calcular e armazenar previamente estas componentes primárias para cada uma abscissa do filtro linear digital da transformada cosseno de 40 pontos, no sentido de minimizar o tempo de processamento do referido método. Dessa forma, para cada abscissa do filtro da transformada cosseno o algoritmo, aqui proposto, limita-se a solucionar numericamente apenas o sistema global de equações lineares, não se detendo em outros cálculos e/ou processamentos. Portanto para o processamento referente ao método do mCSEM $2.5 \mathrm{D}$, aqui proposto, são solucionados apenas 40 sistemas globais de equações lineares complexas, uma vez que as componentes primárias, $\hat{E}_{x}^{p}, \hat{E}_{y}^{p}$ e $\hat{E}_{z}^{p}$, são apenas acessadas automaticamente no percurso do processo.

\section{Validação e/ou calibração das soluções do MCSEM 2.5D \& 1D}

O modelo geoelétrico do mCSEM 2.5D segundo características altamente resistivas em águas profundas, proposto por este trabalho, (Figura 1), é expresso pela seção segundo a perspectiva do plano $z \times x$. Onde a camada da heterogeneidade contendo hidrocarbonetos $(\mathrm{Hc})$ apresenta uma largura na direção $x$ de $50 \mathrm{~km}$, uma espessura $h_{3}$ igual a $50 \mathrm{~m}$ e uma resistividade elétrica $\rho_{3}$ igual a $10 \mathrm{ohm}-\mathrm{m}$, e está a uma profundidade do assoalho oceânico de $950 \mathrm{~m}$. O dipolo elétrico horizontal encontra-se nas coordenadas $x=0.0 \mathrm{~m}$ e $z=1470 \mathrm{~m}$, caracterizado pelo símbolo $\boldsymbol{\nabla}$, e operando à uma freqüência de $0.125 \mathrm{hz}$.

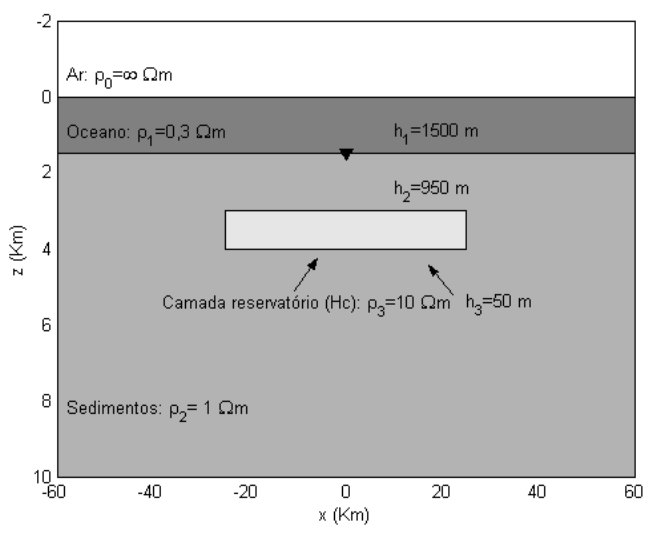

Figura 1 - Seção no plano $z \times x$ referente ao modelo geoelétrico do mCSEM 2.5D com camada reservatório de hidrocarbonetos medindo uma largura de $50 \mathrm{~km}$, na direção $x$.

São apresentadas na Figura 2 as amplitudes, da componente $x$, do campo elétrico em $V A / m^{2}$ referentes ao modelo geoelétrico do mCSEM 1D: $|\mathrm{Ex}|(\mathrm{Hc})$ (modelo 1D com heterogeneidade) e $|\mathrm{Ex}|$ (NoHc) (modelo 1D sem heterogeneidade) e o modelo geoelétrico do mCSEM 2.5D: |Ex| (50Km) (Figura1). 


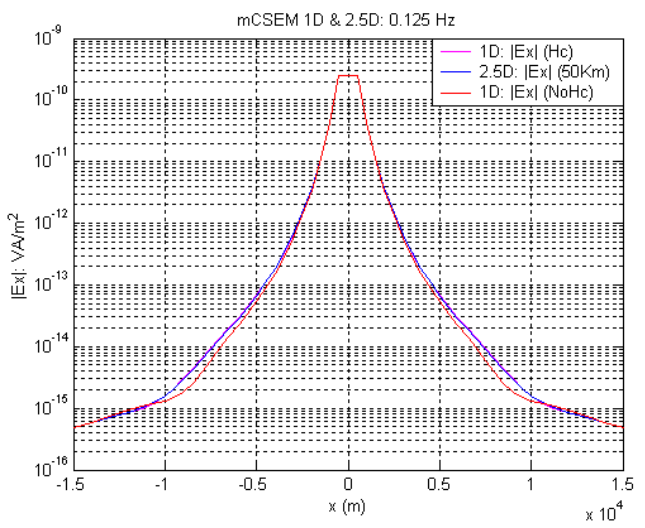

Figura 2 - Respostas 1D, com e sem heterogeneidade contendo hidrocarbonetos $((\mathrm{Hc})$ e (NoHc) respectivamente) e 2.5D (Figura 1) do mCSEM, em termos da amplitude da componente $x$ do campo elétrico.

Agora, temos na Figura 3 as fases, da componente $\mathrm{x}$, do campo elétrico em graus, referentes ao modelo geoelétrico do mCSEM 1D: fase(Ex) (Hc) e fase(Ex) (NoHc) e o modelo geoelétrico do mCSEM 2.5D: fase(Ex) $(50 \mathrm{Km})$ (Figura 1).

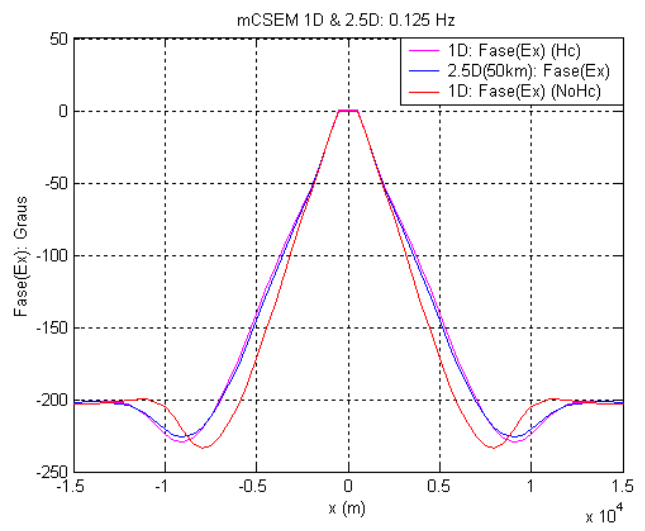

Figura 3 - Respostas 1D, com e sem heterogeneidade contendo hidrocarbonetos $(\mathrm{Hc})$ e $(\mathrm{NoHc})$ e e 2.5D (Figura 1) do mCSEM, em termos da fase da componente $x$ do campo elétrico.

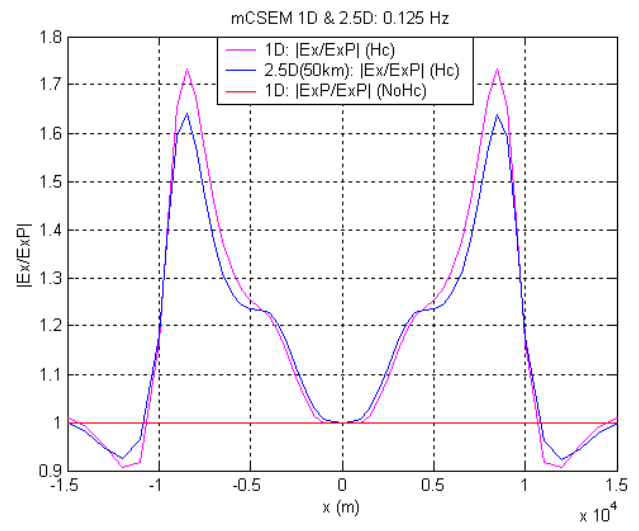

Figura 4 - Respostas 1D e 2.5 do mCSEM, componente $\mathrm{x}$ do campo elétrico, normalizadas pela amplitude da componente $x$ do campo elétrico sem a heterogeneidade com hidrocarbonetos (NoHC).
$\mathrm{Na}$ Figura 4, ao lado, tempos as amplitudes das componentes $x$ do campo elétrico $1 \mathrm{D}$ e $2.5 \mathrm{D}$ do mCSEM, normalizadas pela amplitude da componente $\mathrm{x}$ do campo elétrico primário (|Ex|: ( $\mathrm{NoHc})$ ).

Sensibilidade das soluções do mCSEM 2.5D em relação à variação do comprimento das heterogeneidades

O primeiro modelo geoelétrico do mCSEM 2.5D segundo características altamente resistivas em águas profundas, proposto por Rijo (2005), (Figura 5), é também expresso pela seção segundo a perspectiva do plano $z \times x$. Onde a camada contendo hidrocarbonetos $(\mathrm{Hc})$ apresenta uma largura na direção $x$ de $10 \mathrm{~km}$, uma espessura $h_{3}$ igual a $50 \mathrm{~m}$ e uma resistividade elétrica $\rho_{3}$ igual a 10 ohm-m, e está a uma profundidade do assoalho marinho de $950 \mathrm{~m}$. O dipolo elétrico horizontal encontra-se nas coordenadas $x=0.0 \mathrm{~m}$ e $z=1470 \mathrm{~m}$, caracterizado pelo símbolo $\boldsymbol{\nabla}$, operando à uma freqüência de $0.125 \mathrm{hz}$. Já os três outros modelos geoelétricos do mCSEM 2.5D, também proposto por Rijo (2005), apresentam as camadas contendo hidrocarbonetos $(\mathrm{Hc}) \mathrm{com}$ larguras na direção $x$ de $7 \mathrm{~km}, 5 \mathrm{~km}$ e $3 \mathrm{~km}$, em que estas são as únicas diferenças em relação ao primeiro modelo geoelétrico proposto por Rijo (2005), (Figura 5).

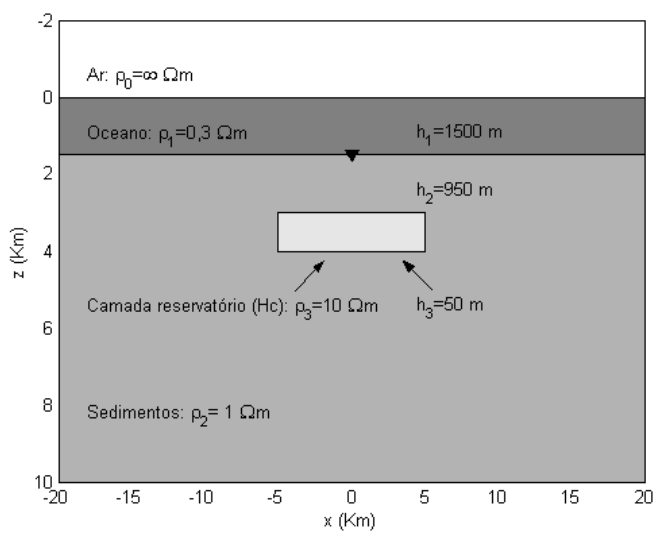

Figura 5 - Seção no plano $z \times x$ referente ao modelo geoelétrico do mCSEM $2.5 \mathrm{D}$ com camada reservatório de hidrocarbonetos medindo uma largura de $10 \mathrm{~km}$, na direção $x$.

Nas Figuras (6)-(8), temos as soluções (do mCSEM 2.5D e 1D) das amplitudes, fases e amplitudes normalizadas, da componente $\mathrm{x}$ do campo elétrico, respectivamente, referentes aos modelos geoelétricos propostos por Rijo (2005), e descritos acima. 


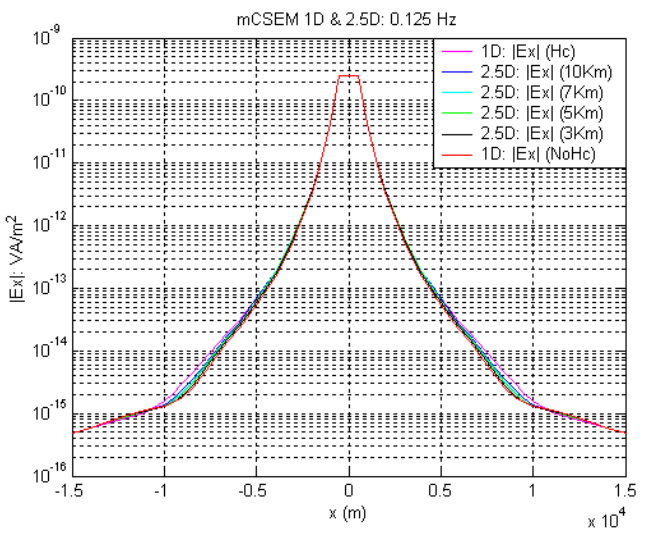

Figura 6 - Soluções 1D e 2.5 do mCSEM, das amplitudes da componente $\mathrm{x}$ do campo elétrico, a partir dos modelos geoelétricos descritos acima e também propostos por Rijo (2005).

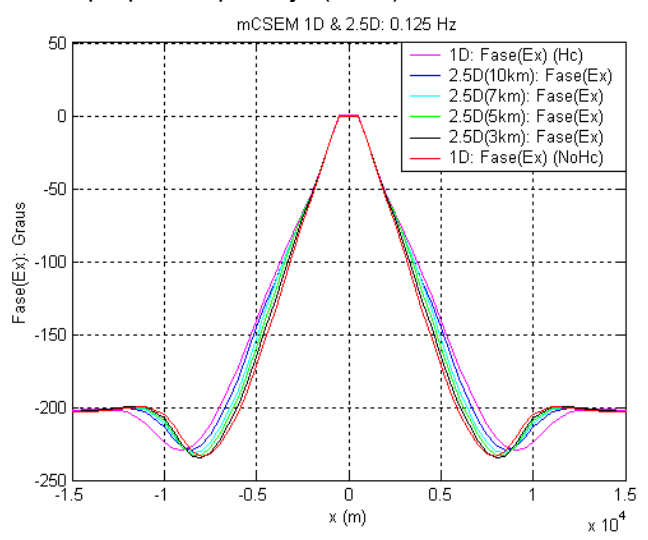

Figura 7- Soluções 1D e 2.5 do mCSEM, das fases da componente $\mathrm{x}$ do campo elétrico, a partir dos modelos geoelétricos descritos acima e também propostos por Rijo (2005).

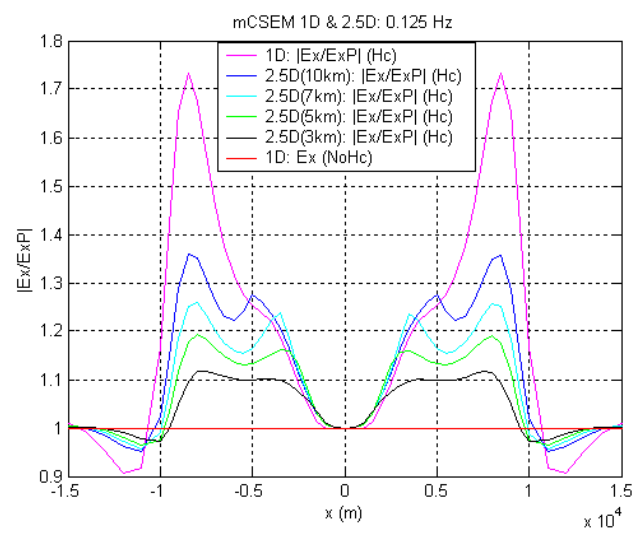

Figura 8- Soluções 1D e 2.5 do mCSEM, amplitudes das componentes $\mathrm{x}$ do campo elétrico, normalizadas pela amplitude da componente $\mathrm{x}$ do campo elétrico sem hidrocarbonetos (NoHC).

\section{Conclusões}

As respostas do mCSEM 2.5D (Figuras 2-4), apresentam formas e aproximações coerentes em relação às resposta do mCSEM 1D (Figuras $2-4)$, ou seja, elas tendem às respostas do mCSEM 1D (Figuras 2-4). As soluções do mCSEM 2.5D (Figuras 6-8) apresentam sensibilidade em relação à variação da largura na direção $\mathrm{x}$ da heterogeneidade, similar às soluções apresentadas por Rijo (2005).

Cada uma das soluções do mCSEM 2.5D propostas, apresentaram tempo de processamento em média de 11,5 segundos, em um PC com CPU 2,83 GHz, 3,46 GB de RAM e administrado por um sistema operacional Windows XP 2002. Para inversão geofísica EM, a metodologia proposta, que minimiza o tempo de processamento da modelagem direta do mCSEM 2.5D, pode ser aplicada. Desde que, se escolha a vizinhança de interesse contendo a hipotética heterogeneidade para que se calcule e armazene previamente as componentes primárias da fonte existentes nesta vizinhança.

\section{Agradecimentos}

In memoriam do professor Luiz Rijo e de meu pai.

\section{Referências}

Almeida, F. L., 2002. Filtros otimizados para as transformadas seno, co-seno e de Hankel J0, J1 e J2. Dissertação (mestrado em geofísica) Centro de Geociências, Universidade Federal do Pará. 110 p.

Burden, R. L.; Faires, J. D., 2008. Análise Numérica. $8^{a}$ edição. Cengage Learning.

Jin, J., 2002. The Finite Element Method in Electromagnetics. Second Edition. Johm Wiley \& Sons, New York.

Rijo, L., 2007. Cooperação UniversidadeIndústria: O caso do método marine Controlled Source Electromagenetic (CSEM) na exploração de hidrocarbonetos. $10^{\text {th }}$ International Congress of the Brazilian Geophysical Society held in Rio de Janeiro, Brazil. 1 CD-ROM.

Rijo, L., 2005. 2.5 - D Finite Element Algorithm for MCSEM Modeling. $9^{\text {th }}$ International Congress of the Brazilian Geophysical Society held in Salvador, Brazil.

Rijo, L., 2002. Teoria dos Métodos Eletromagnético I, II e III - Notas de aula. Departamento de Geofísica, UFPa.

Silva, H. F., 2012. MODELAGEM NUMÉRICA DE DADOS MCSEM 2.5D. Dissertação de Mestrado, UFPA, $63 p$

Souza, V. C. T. 2007. Modelagem numérica de dados MCSEM 3D usando computação paralela. Tese de Doutorado, UFPA. $110 \mathrm{p}$.

Souza, V. C. T., Rijo, L. e Silva, M. W. C., 2005. The preconditioned biconjugate gradient algorithm applied to geophysical electromagnetic modeling. $9^{\text {th }}$ International Congress of the Brazilian Geophysical Society held in Salvador, Brazil.

Zienkienwicz, O. C., Taylor, R. L., 2000. The finite element method - Volume 1: the basis. Oxford: Butterworth Heinemann. 